\title{
Differences in Sympathetic Nervous Stimulation of Brown Adipose Tissue Between the Young and Old, and the Lean and Obese
}

\author{
Lonneke Bahler $^{1}$, Hein J. Verberne ${ }^{2}$, Wanda M. Admiraal ${ }^{1}$, Wim J. Stok ${ }^{3}$, Maarten R. Soeters ${ }^{4}$, Joost B. Hoekstra ${ }^{1}$, \\ and Frits Holleman ${ }^{1}$ \\ ${ }^{I}$ Department of Internal Medicine, Academic Medical Center, Amsterdam, The Netherlands; ${ }^{2}$ Department of Nuclear Medicine, \\ Academic Medical Center, Amsterdam, The Netherlands; ${ }^{3}$ Laboratory for Clinical Cardiovascular Physiology, Center for Heart \\ Failure Research, Academic Medical Center, Amsterdam, The Netherlands; and ${ }^{4}$ Department of Endocrinology and Metabolism, \\ Academic Medical Center, Amsterdam, The Netherlands
}

\begin{abstract}
Brown adipose tissue (BAT) could facilitate weight loss by increasing energy expenditure. Cold is a potent stimulator of BAT, activating BAT primarily through the sympathetic nervous system (SNS). Older or overweight individuals have less metabolic BAT activity than the lean and young, but the role of the SNS in this decline is unknown. We aimed to determine whether this lower metabolic BAT activity in older or overweight individuals can be explained by a lower SNS response to cold. Methods: This was a prospective observational study. We included 10 young obese, 11 old lean, and 14 young lean healthy men. All subjects underwent ${ }^{18} \mathrm{~F}-\mathrm{FDG}$ PET/CT and ${ }^{123}$-meta-iodobenzylguanidine (123/-mIBG) SPECT/CT after an overnight fast and $2 \mathrm{~h}$ of cold exposure. Metabolic BAT activity was expressed as volume and as SUV $_{\max }$ of ${ }^{18} \mathrm{~F}$-FDG. BAT SNS activity was expressed as volume and as the ratio between ${ }^{123} \mathrm{I}-\mathrm{mIBG}$ uptake in BAT and a reference region (SQUV $_{\max }$ of $\left.{ }^{123} \mid-m I B G\right)$. Results: SUV $_{\max }$, BAT volume, and SQUV $V_{\max }$ were significantly different between young and old (SUV $V_{\max }, 7.9$ [range, 4.2-17.3] vs. 2.9 [range, 0.0-4.0]; volume, 124.8 [range, 10.9-338.8] vs. 3.4 [range, 0.0-10.9]; and SQUV ${ }_{\max }, 2.7$ [range, 1.9-4.7] vs. 0.0 [range, 0.0-2.2], respectively) (all $P<0.01$ ) but not between lean and obese $\left(\mathrm{SUV}_{\max }, 7.9\right.$ [range, 4.2-17.3] vs. 4.0 [range, 0.0-13.5] $[P=0.69$ ]; volume, 124.8 [range, 10.9-338.8] vs. 11.8 [range, 0.0-190.2] $[P=$ 0.64 ]; and $S Q U V_{\max }, 2.7$ [range, 1.9-4.7] vs. 1.7 [range, 0-3.5] $[P=$ 0.69 ], respectively). We found a strong positive correlation between BAT activity measured with ${ }^{18} \mathrm{~F}-\mathrm{FDG}$ and ${ }^{123} \mathrm{I}-\mathrm{mIBG}$ in the whole group of BAT-positive subjects $(\rho=0.82, P<0.01$ ). Conclusion: Both sympathetic drive and BAT activity are lower in older but not in obese men.
\end{abstract}

Key Words: brown adipose tissue; ${ }^{18} \mathrm{~F}-\mathrm{FDG}$ PET/CT; ${ }^{123} \mathrm{I}-\mathrm{mIBG}$ SPECT/CT; age; obesity

J Nucl Med 2016; 57:372-377

DOI: 10.2967/jnumed.115.165829

$\mathbf{T}$ he increasing prevalence of obesity in the young heralds a period in which we will be confronted with an older and obese population with an abundance of adverse consequences and associated health

Received Jul. 31, 2015; revision accepted Oct. 28, 2015.

For correspondence or reprints contact: Lonneke Bahler, Academic Medical Center, Department of Internal Medicine F4-255, P.O. Box 22660, 1100DD Amsterdam, The Netherlands.

E-mail: I.bahler@amc.uva.nl

Published online Nov. 25, 2015.

COPYRIGHT (c) 2016 by the Society of Nuclear Medicine and Molecular Imaging, Inc. costs $(1,2)$. So far most solutions for this problem have been unsatisfactory because attempts to lose weight fail in most cases $(3,4)$.

Metabolically active brown adipose tissue (BAT) increases energy expenditure and could play a role in the battle against obesity by facilitating weight loss $(5,6)$. If we could identify factors that contribute to stimulation or recruitment of metabolically active BAT, it would be possible to use BAT as a means to lose weight. Though cold exposure is the strongest activator of BAT known so far (7), people will not be exposed to cold for most of the day. Factors activating BAT during thermoneutrality have to be found. An important target in this respect is the sympathetic nervous system (SNS), which is thought to be the primary activator of BAT (8).

In both older and overweight people, metabolic activity of BAT is generally found to be much lower than in younger or leaner controls (9-12). The reason for this decreased metabolic BAT activity remains unknown, and the role of the SNS in BAT activation in the older and the obese has not been studied.

The common method to demonstrate metabolically active BAT is by performing ${ }^{18} \mathrm{~F}$-FDG PET/CT. Recently, our group showed that the sympathetic nervous stimulation of BAT can be visualized by ${ }^{123}$ I-meta-iodobenzylguanidine ( $\left.{ }^{123} \mathrm{I}-m \mathrm{IBG}\right)$ SPECT (13). We showed a strong, positive correlation between ${ }^{18} \mathrm{~F}-\mathrm{FDG}$ and ${ }^{123} \mathrm{I}-$ $m$ IBG in BAT in lean young men.

We hypothesized that SNS activation is diminished in both older and obese subjects as compared with young lean subjects as a cause for the diminished BAT activity. Therefore, we investigated whether SNS activation of BAT ( ${ }^{123} \mathrm{I}-\mathrm{mIBG}$ ) as a mediator of BAT activity $\left({ }^{18} \mathrm{~F}-\mathrm{FDG}\right)$ is diminished in young obese or lean older men when compared with the lean and young. In addition, we investigated whether the strong correlation between ${ }^{18} \mathrm{~F}-\mathrm{FDG}$ and ${ }^{123} \mathrm{I}-m \mathrm{IBG}$ in lean men is also present in obese and older men. Finally, we investigated whether energy expenditure correlated with BAT activity.

\section{MATERIALS AND METHODS}

This study was approved by the Medical Ethics Committee of the Academic Medical Center of the University of Amsterdam and was conducted according to the Declaration of Helsinki. Written informed consent was obtained from all subjects after oral and written explanation of the study procedures. The registration numbers of this study are NCT02173834 and NCT02130154 (www.clinicaltrials.gov).

\section{Participants}

This study consists of 2 separate protocols, which we have combined into 1 study. Power calculations were performed using nQuery Advisor 
(Statistical Solutions Ltd.), and they were based on previous studies (13). Assuming an $\alpha$ of 5\%, 10 subjects per group were needed to be included in the study to obtain a power of $80 \%$ to detect a significant difference between the young obese and young lean subjects. To detect a significant difference between the young lean and old lean subjects, assuming an $\alpha$ of 5\%,11 subjects per group were needed to be included in the study to obtain a power of $80 \%$ to detect a significant difference between the young lean and old lean subjects.

We decided to combine the 2 studies after inclusion of 14 young lean subjects. In all, we included a group of 35 healthy, Caucasian male volunteers. We studied 10 young obese men (inclusion criteria: age, 18-30 y; body mass index [BMI], $>28 \mathrm{~kg} / \mathrm{m}^{2}$; later referred to as the obese men), 11 old lean men (inclusion criteria: age, $>40 \mathrm{y}$; BMI, $19-25 \mathrm{~kg} / \mathrm{m}^{2}$; later referred to as the older men), and 14 lean young men (inclusion criteria: age, $18-30 \mathrm{y}$; BMI, $19-25 \mathrm{~kg} / \mathrm{m}^{2}$; later referred to as lean young). Subjects were included when they met the age and BMI criteria of the specified group and did not use any medication or drugs.

Baseline characteristics were obtained during the first visit. Anthropometric data were measured with the subjects wearing only underwear. Weight was measured on the same calibrated mechanical scale (SECA) to the nearest $100 \mathrm{~g}$, and height and waist circumference were recorded to the nearest $0.01 \mathrm{~m}$.

\section{${ }^{18}$ F-FDG PET/CT and ${ }^{123}$ I-mIBG SPECT/CT \\ Imaging Protocols}

${ }^{18} \mathrm{~F}-\mathrm{FDG}$ PET/CT and ${ }^{123} \mathrm{I}-\mathrm{mIBG}$ SPECT/CT were performed on 2 separate days within an interval of $2 \mathrm{wk}$ between April 2013 and March 2014 according to the previously established protocol (14). In short, all subjects arrived in the morning after an overnight fast, after which they were exposed to mild cold $\left(\sim 16^{\circ} \mathrm{C}-17^{\circ} \mathrm{C}\right)$ in an air-cooled room for $2 \mathrm{~h}$. During the cold exposure, subjects were wearing underwear only. After $1 \mathrm{~h}$ of cold exposure, the radioactive tracer was administered (the ${ }^{18} \mathrm{~F}-\mathrm{FDG}$ was adjusted for BMI, leading to dosages of $\sim 200 \mathrm{MBq}$; for ${ }^{123} \mathrm{I}-m \mathrm{IBG}$, a fixed dose of $185 \mathrm{MBq}$ was used). ${ }^{18} \mathrm{~F}-\mathrm{FDG}$ PET/CT was performed directly after the 2-h cold exposure (i.e., $1 \mathrm{~h}$ after ${ }^{18} \mathrm{~F}-\mathrm{FDG}$ administration), and ${ }^{123} \mathrm{I}-m \mathrm{IBG}$ SPECT/CT was performed approximately $24 \mathrm{~h}$ after the ${ }^{123} \mathrm{I}-m \mathrm{IBG}$ administration (according to the previously established protocol) (13). After every 2 subjects, the sequence of ${ }^{18} \mathrm{~F}-\mathrm{FDG}$ PET/CT and ${ }^{123} \mathrm{I}-m \mathrm{IBG}$ SPECT was changed to overcome order effects.
The scans were assessed by 2 authors, one trained in analyzing the scans and the other a nuclear physician. To compare areas with active BAT on both scans, ${ }^{18} \mathrm{~F}-\mathrm{FDG}$ PET/CT and ${ }^{123} \mathrm{I}-m \mathrm{IBG}$ SPECT/CT were aligned. Volumes of interest established on ${ }^{18}$ F-FDG PET/CT (i.e., uptake of ${ }^{18} \mathrm{~F}-\mathrm{FDG}$ identified by PET and the presence of fat identified by $\mathrm{CT}$ ) were copied to the aligned ${ }^{123} \mathrm{I}-m$ IBG SPECT/CT images. PET and SPECT images were aligned using an automated rigid method. We measured maximal uptake, mean uptake, and volume of BAT on both ${ }^{18} \mathrm{~F}$-FDG PET/CT and ${ }^{123} \mathrm{I}-m \mathrm{IBG}$ SPECT/CT.

BAT volume was measured using a PET- or SPECT-threshold-based delineation. Therefore, volumes measured reflect activated BAT volume. All visually identified areas with active BAT were included in the analysis.

To be able to integrate the influence of both the metabolic and the sympathetic BAT activity within subjects across the 3 groups, we also calculated the SQUV $\mathrm{max}_{\text {max }}$-to-SUV $\mathrm{Smax}_{\operatorname{mat}}$ ratio.

\section{Other Measurements}

Energy expenditure was recorded every minute using Quark RMR (Cosmed) during $30 \mathrm{~min}$ via indirect calorimetry after the $2 \mathrm{~h}$ of cold exposure in the waiting period after ${ }^{123} \mathrm{I}-m \mathrm{IBG}$ administration and the SPECT/CT acquisition (e.g., $1 \mathrm{~h}$ after the ${ }^{123} \mathrm{I}-\mathrm{mIBG}$ administration).

Oral glucose tolerance test and laboratory measurements were performed according to standard procedures (the supplemental data provide detailed methods; supplemental materials are available at http:// jnm.snmjournals.org).

\section{Statistical Analysis}

The characteristics of the study participants are reported as medians with interquartile ranges (IQRs). The $P$ values for differences between the lean young men and the older men and the obese were determined using the Mann-Whitney $U$ test. Differences in the presence of BAT activity between the groups were calculated with the Fisher exact test. Correlations were determined with the Spearman correlation. Data analysis was performed using SPSS software 20.0 (IBM Software). A $P$ value of less than 0.05 was considered to indicate statistical significance.

\section{RESULTS}

The study was performed between April 2013 and March 2014. Baseline characteristics of the subjects are shown in Table 1

TABLE 1

Characteristics of Male Volunteers

\begin{tabular}{|c|c|c|c|c|c|c|}
\hline Characteristic & $\begin{array}{l}\text { Obese } \\
\text { young }\end{array}$ & IQR & $\begin{array}{l}\text { Lean } \\
\text { young }\end{array}$ & IQR & $\begin{array}{l}\text { Lean } \\
\text { old }\end{array}$ & IQR \\
\hline$N$ & 10 & & 14 & & 11 & \\
\hline Age (y) & 25.5 & $21.0-31.3$ & 25.5 & $20.8-28.0$ & $54^{*}$ & $50.0-60.0$ \\
\hline BMI $\left(\mathrm{kg} / \mathrm{m}^{2}\right)$ & $32.2^{*}$ & $30.8-38.9$ & 22.0 & $20.9-23.2$ & 23.1 & $21.7-23.8$ \\
\hline Waist circumference (cm) & $117.5^{\star}$ & $110.5-144.5$ & 83.0 & $82.0-85.0$ & $90.0^{\dagger}$ & $85.0-93.0$ \\
\hline \multicolumn{7}{|l|}{ Laboratory values } \\
\hline $\mathrm{HbA} 1 \mathrm{c}(\mathrm{mmol} / \mathrm{mol})$ & 35.0 & $32.8-37.8$ & 33.5 & $31.8-35.0$ & $39.0^{\star}$ & $36.0-40.0$ \\
\hline $\begin{array}{l}\text { Thyroid-stimulating } \\
\text { hormone (mE/L) }\end{array}$ & 2.4 & $1.5-3.3$ & 1.8 & $1.0-2.4$ & 1.7 & $1.4-2.2$ \\
\hline Free fatty acids (mmol/L) & 0.6 & $0.6-0.8$ & 0.5 & $0.4-0.7$ & 0.5 & $0.3-0.8$ \\
\hline $\begin{array}{l}\text { Energy expenditure in } \\
\text { cold (kcal/24 h) }\end{array}$ & 2,421 & $1,499-2,530$ & 2,294 & $\begin{array}{l}1,807- \\
2,666\end{array}$ & 1,484 & $\begin{array}{l}1,033- \\
2,294\end{array}$ \\
\hline
\end{tabular}

${ }^{\star} P<0.001$, significantly different as compared with lean young men.

$\dagger P<0.01$, significantly different as compared with lean young men.

Data are $n$ or median. 
(a more detailed table of baseline characteristics can be found in the supplemental data). As expected, the obese and the older men showed a more adverse metabolic profile than the lean and young subjects; that is, older subjects had a significantly higher HbA1c and total and low-density lipoprotein cholesterol whereas obese subjects were significantly less insulin-sensitive and had higher high-density lipoprotein cholesterol values and triglycerides (Supplemental Table 1).

${ }^{18}$ F-FDG uptake was visually seen in 7 of 10 subjects in the obese-male group, 6 of 11 in the older-male group, and 13 of 14 in the lean-young-male group. The presence of BAT activity on ${ }^{18} \mathrm{~F}$ FDG PET/CT was not significantly different between the obese and lean males $(P=0.27)$ and borderline significant between the old and young males $(P=0.056)$. Figure 1 shows 3 typical cases of metabolically active BAT in our subjects. Metabolic BAT activity (maximal and mean) and volume measured on ${ }^{18} \mathrm{~F}-\mathrm{FDG}$ PET/CT were different between the lean older men and lean young $(P<$ 0.01) men but not between the obese and lean young men (Fig. 2).

Uptake of ${ }^{123}$ I-mIBG was seen in 7 of 10 subjects in the obesemale group, 5 of 11 in the older-male group, and 13 of 14 in the lean-young-male group. Again, the presence of BAT activity on ${ }^{123}$ I- $m$ IBG SPECT was not significantly different between the obese and lean men. The maximal sympathetic nervous stimulation of BAT $\left(\mathrm{SQUV}_{\max }\right)$ and volume measured on ${ }^{123} \mathrm{I}-m \mathrm{IBG}$ SPECT were significantly diminished in the older men as compared with the lean young but did not differ between the obese and the lean young men. Mean sympathetic nervous stimulation of BAT ( $\mathrm{SQUV}_{\text {mean }}$ ) was not significantly different between any of the groups (Fig. 2).

All the subjects with ${ }^{123} \mathrm{I}-m \mathrm{IBG}$ uptake in BAT also showed ${ }^{18} \mathrm{~F}$ FDG uptake in BAT. Conversely, there was 1 older subject who showed ${ }^{18} \mathrm{~F}$-FDG uptake in BAT but no ${ }^{123} \mathrm{I}-m \mathrm{IBG}$ uptake.

To assess the relative influence of the sympathetic drive, we calculated the maximal and mean SQUV/SUV ratio in all subjects who were BAT-positive on both scans. Although both the SQUV $\mathrm{max}_{\max }$ and the $\mathrm{SUV}_{\max }$ were lower in the older men, the SQUV $\mathrm{max}_{\max }-$ to-SUV $_{\max }$ ratio was higher in the older men than the lean young. There was no difference in the ratio between the obese and the lean young. The $\mathrm{SQUV}_{\text {mean }} / \mathrm{SUV}_{\text {mean }}$ ratio did not differ between the groups (Fig. 3).

There was a negative correlation between age and maximal metabolic and sympathetic BAT activity. Although the correlation

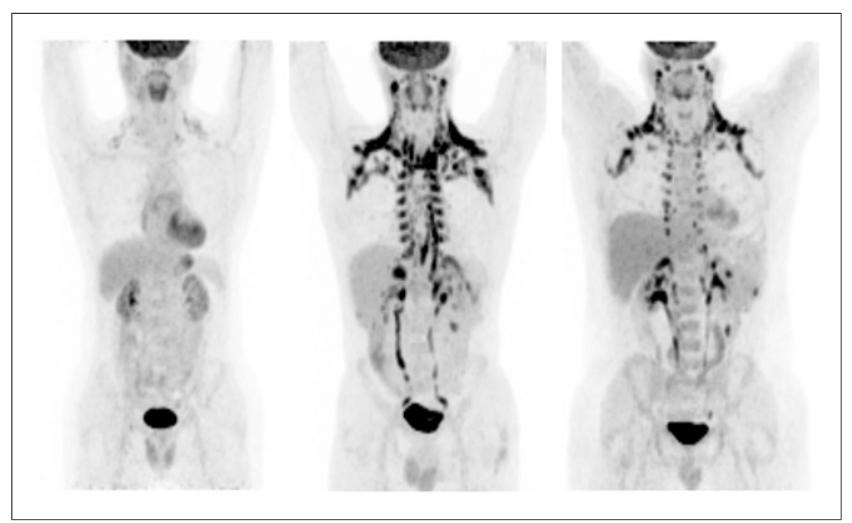

FIGURE 1. Three typical examples of BAT activity in accordance with subjects' age and BMI. Left subject is a lean and old subject showing minimal BAT activity; middle subject is a lean and young subject showing abundant BAT activity; and right subject is an obese and young subject showing BAT activity but less than lean and young subject.

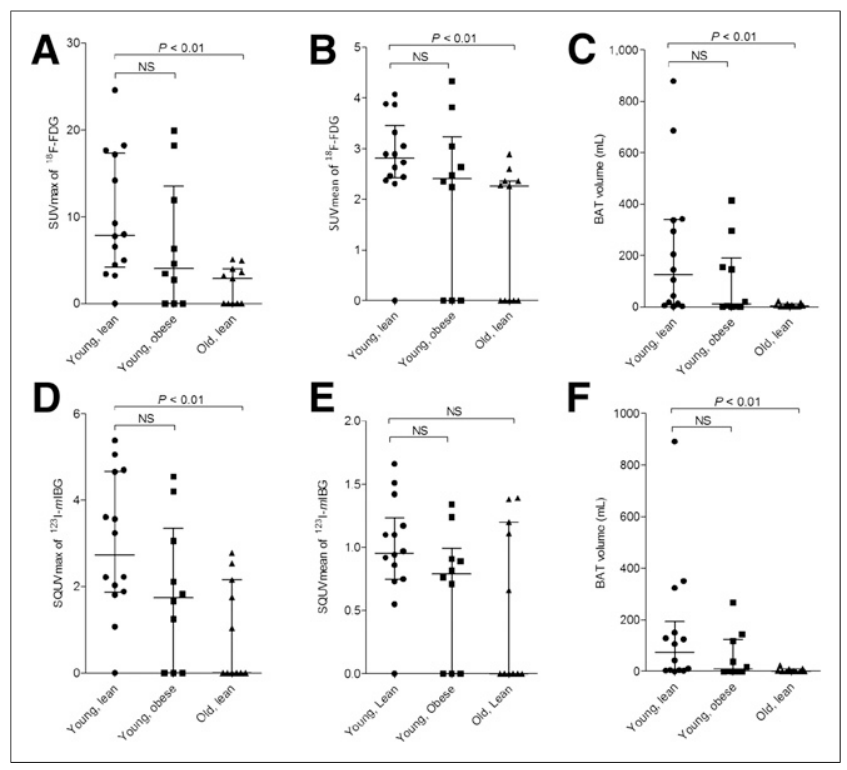

FIGURE 2. (A-C) Metabolic activity of BAT between the 3 groups defined as median (IQR) SUV ${ }_{\text {max }}, S_{\text {SU }}$ mean, and volume of ${ }^{18} \mathrm{~F}-\mathrm{FDG}$ (defined as activity in $\mathrm{Bq} / \mathrm{mL}$ within region of interest divided by injected dose in Bq/g of body weight), respectively. (D-F) Sympathetic nervous stimulation to BAT between the 3 groups defined as median (IQR) SQUV $_{\text {max }}$ and volume of ${ }^{123}$ - $m$ IBG (calculated as maximum count in BAT divided by mean count per voxel in the mediastinum), respectively. NS $=$ not significant.

remained significant for age and mean metabolic BAT activity, the correlation disappeared for age and the mean sympathetic BAT activity (Table 2). There was no correlation between BMI and maximal or mean metabolic and maximal sympathetic BAT activity or BMI and BAT volume (Table 2). There was a strong and positive correlation in the whole group for both maximal and mean BAT activity measured with ${ }^{18} \mathrm{~F}-\mathrm{FDG}$ and ${ }^{123} \mathrm{I}-m \mathrm{IBG}$ (Fig. 4). Also after removal of the BAT-negative subjects, this correlation remained strong and positive for maximal BAT activity but not for mean BAT activity (Fig. 4).

After stratification, the strong positive correlation between maximal BAT activity measured with ${ }^{18} \mathrm{~F}$-FDG and ${ }^{123} \mathrm{I}-m \mathrm{IBG}$ was still apparent in the lean young and the obese men but not in the older men (Table 3). After removal of the BAT-negative subjects, the correlation was still significant in the lean young men (spearman correlation $\rho, 0.74 ; P<0.01$ ) but not for the obese men (spearman correlation $\rho, 0.12 ; P=0.64$ ). There was no correlation between mean BAT activity measured with ${ }^{18} \mathrm{~F}$-FDG and ${ }^{123} \mathrm{I}-\mathrm{mIBG}$ for any of the groups (Table 3).

Energy expenditure during cold did not differ significantly among the 3 groups (Table 1). There was a positive relation between energy expenditure in cold and maximal metabolic and sympathetic BAT activity in the whole group (Table 2).

\section{DISCUSSION}

We found a lower SNS activity in the older men than in the lean young men, which may be the explanation for the lower metabolic BAT activity. In line with our earlier data, we found a strong and positive correlation for detecting BAT on ${ }^{18} \mathrm{~F}$-FDG PET/CT and ${ }^{123} \mathrm{I}-m$ IBG SPECT/CT in the lean young men (13). This correlation disappeared in the older men. 


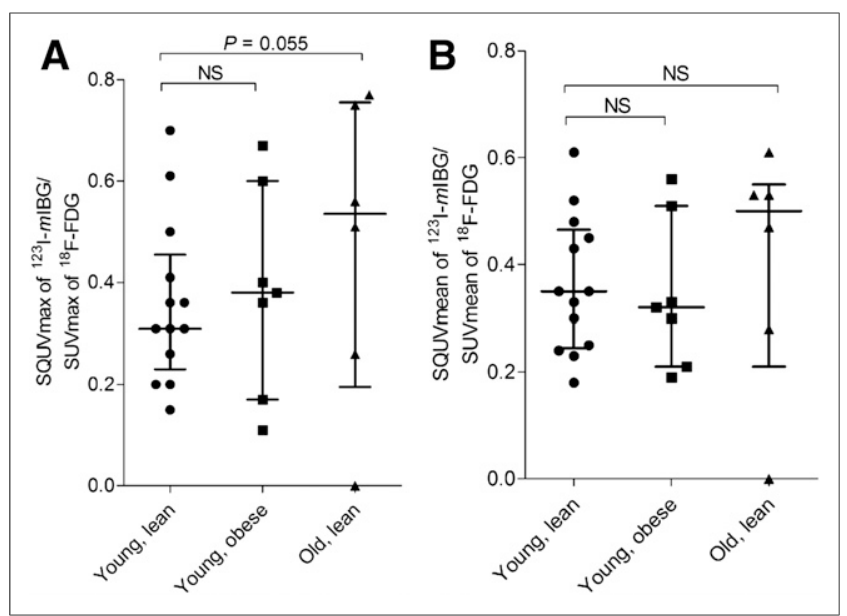

FIGURE 3. Relative influence of sympathetic drive, calculated as SQUV-to-SUV ratio in all subjects who were BAT-positive on both scans for maximal values $(A)$ and mean values $(B) . N S=$ not significant.

The higher SQUV $\mathrm{max}_{\max }$-to-SUV $\mathrm{S}_{\max }$ ratio in the older men than in lean young men may suggest a dissociation between the stimulus and effect. This dissociation might also explain the absence of a correlation for detecting BAT between ${ }^{18} \mathrm{~F}$-FDG PET/CT and ${ }^{123} \mathrm{I}-$ $m$ IBG SPECT/CT.

Because the SNS drive is supposed to be the main determinant of BAT activity, an increasing ratio of SNS activity over metabolic BAT activity (for our purposes the ratio of ${ }^{123} \mathrm{I}-m \mathrm{IBG}$ uptake over ${ }^{18} \mathrm{~F}$ FDG uptake) might imply that BAT becomes less sensitive to SNS signals on aging. After all, the SNS signal, relative to metabolic activity (i.e., the $\mathrm{SQUV}_{\max }$-to-SUV $\mathrm{SWx}_{\max }$ ratio) was higher in the older men than in the lean young men, though just short of statistical significance.

The combination of both a lower absolute SNS signal and a possible declined sensitivity of BAT for SNS stimulation may cause the lower ability to activate and recruit BAT in the older men. However, the relative role of the SNS should be confirmed in trials with larger subject numbers.

Strikingly, older humans have an inability to appropriately regulate their temperature when exposed to cold (15-17). At least some part of this inability is due to an increased heat loss caused by inefficient vasoconstriction responses or changes in peripheral blood flow during cold exposure (17-19). Nonetheless, there is also evidence that on aging, the ability to produce metabolic heat decreases (20). Hypothetically, this might at least in part be caused by the lower absolute SNS signal to BAT in the elderly population, causing a lower ability to activate and recruit BAT in the older men.

These findings support the idea that the SNS has an important role in the activation process of BAT.

Studies investigating the effects of sympathicomimetics (e.g., ephedrine, isoprenaline) on BAT activation did not result in unequivocal evidence (21-24). However, recently Cypess et al. showed an intense BAT activation after administration of mirabegron (a $\beta 3$ adrenergic receptor agonist) (25). Similar to our previous study (13), we also clearly show involvement of SNS in BAT. The SNS activity to BAT can be visualized and quantified by performing ${ }^{123}$ I- $m$ IBG SPECT.

The involvement of the SNS in BAT activation is clearly demonstrated by the strongly positive correlation for detecting BAT on ${ }^{123} \mathrm{I}-m$ IBG SPECT and ${ }^{18}$ F-FDG PET/CT in lean young, healthy men (13). The lower metabolic BAT activity in the older men than in the lean young men is in line with previous studies, though less pronounced $(10,12)$. We found a fairly high metabolic BAT activity in the older men as compared with the literature; this might be explained by the fact that the older men in our study were in a good physical condition as illustrated by a relatively low BMI and the fact that none of our subjects used any medication, despite the median age of $54 \mathrm{y}$ (IQR; range, 50-60 y). We found a borderline significant difference in the presence of BAT activity on ${ }^{18}$ F-FDG PET/CT between the young and older men. Though the metabolic BAT activity in the older men was relatively high, the borderline significant difference is probably a type 2 error, caused by the small sample size.

In addition to the lower metabolic BAT activity in the older men, we now demonstrate a lower SNS activity to BAT, as compared with the lean, young. This might, at least in part, explain the lower metabolic BAT activity in older men. Promising results have been obtained in several studies indicating that BAT can be recruited, even in individuals with decreased BAT activity $(26,27)$. However, whether this could also be applied in older humans has to be investigated.

We used ${ }^{123} \mathrm{I}-m$ IBG SPECT to measure sympathetic stimulation in an acute exposure setting. The uptake of ${ }^{123} \mathrm{I}-\mathrm{mIBG}$ in neuroendocrine cells occurs by an active uptake mechanism via the norepinephrine transporter. There is evidence that acute modulation of norepinephrine transporter trafficking may provide additional modulatory capacity for noradrenergic signaling (28). This means that the ${ }^{123} \mathrm{I}-\mathrm{mIBG}$ uptake patterns we have observed most likely reflect the fast sympathetic response to the acute cold exposure. After the uptake, ${ }^{123} \mathrm{I}-m \mathrm{IBG}$ is stored in neurosecretory granules and may subsequently be secreted in the synaptic cleft.

TABLE 2

Correlations Between BAT Parameters and Patient Characteristics

\begin{tabular}{lllllcc}
\hline \multicolumn{1}{c}{ Characteristic } & SUV $_{\text {max }}$ & SUV $_{\text {mean }}$ & SQUV $_{\text {max }}$ & SQUV $_{\text {mean }}$ & BAT volume & BAT volume \\
\hline Age $(\mathrm{y})$ & $\rho=-0.48^{*}$ & $\rho=-0.44^{\star}$ & $\rho=-0.38^{\dagger}$ & $\rho=-0.272$ & $\rho=-0.43^{\star}$ & $\rho=-0.37^{\dagger}$ \\
$\mathrm{BMI}\left(\mathrm{kg} / \mathrm{m}^{2}\right)$ & $\rho=-0.26$ & $\rho=-0.28$ & $\rho=-0.27$ & $\rho=-0.29$ & $\rho=-0.26$ & $\rho=-0.28$ \\
Energy expenditure in cold $(\mathrm{kcal} / 24 \mathrm{~h})$ & $\rho=0.47^{\dagger}$ & $\rho=0.48^{\dagger}$ & $\rho=0.46^{\dagger}$ & $\rho=0.41$ & $\rho=0.55^{\dagger}$ & $\rho=0.41$
\end{tabular}

${ }^{\star} P<0.01$.

${ }^{\dagger} P<0.05$

Spearman correlations for BAT activity parameters and age, BMI, and energy expenditure in cold. 


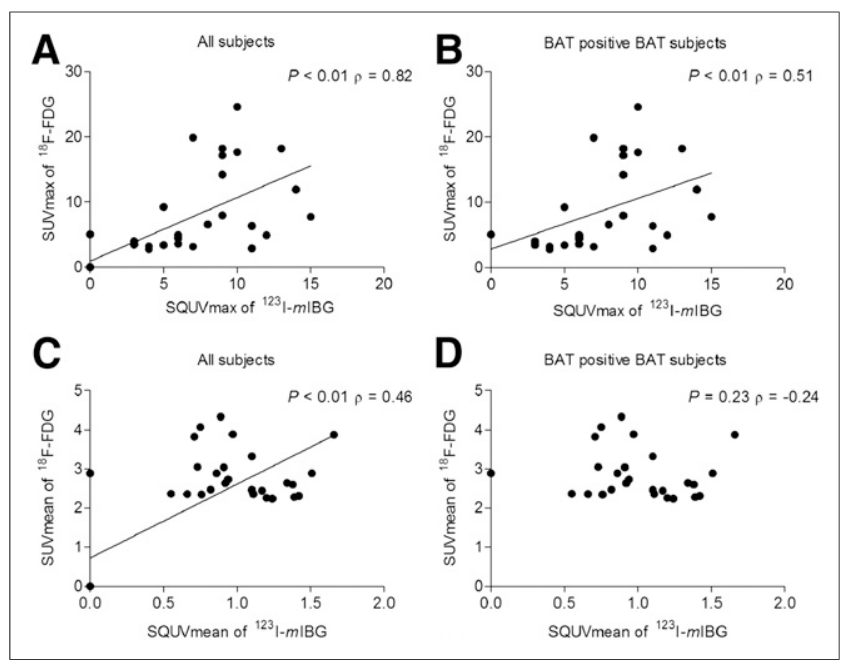

FIGURE 4. Spearman correlations for metabolic BAT activity measured on ${ }^{18} \mathrm{~F}-\mathrm{FDG}$ PET/CT (expressed as SUV $\mathrm{max}_{\max }$ of ${ }^{18} \mathrm{~F}-\mathrm{FDG}$ ) and sympathetic nervous stimulation to BAT measured on ${ }^{123}$ - $m$ IBG SPECT (expressed as SQUV max $_{\text {ax }}$ of ${ }^{123} \mid-m I B G$ ). (A) Maximal BAT activity in all subjects $(n=35)$. (B) Maximal BAT activity in all BAT-positive subjects $(n=26)$. (C) Mean BAT activity in all subjects $(n=35)$. (D) Mean BAT activity in all BAT-positive subjects $(n=26)$; regression line not shown because of nonsignificance.

${ }^{123} \mathrm{I}-m$ IBG uptake in a specific organ is dependent on catecholamine excretion or the adrenergic innervation of the specific organ (29).

In contrast to other groups, we used a uniform cooling method in which all subjects were exposed to the same room temperature instead of personalized cooling. Uniform cooling is considered clinically more relevant because humans are exposed to outdoor temperatures, which are also uniform. Nonetheless, despite the uniform cooling, we found no differences in metabolic BAT activity between the lean young and the obese men. This might be explained by the slightly lower age of the obese subjects than the subjects in the literature $(30,31)$. Aging processes might be more important as determinants of BAT than BMI (9). Furthermore, the small number of participants may have caused a lack of power to demonstrate a

TABLE 3

Correlations Between BAT Parameters After Stratification

\begin{tabular}{|c|c|c|}
\hline Characteristic & $S U V_{\max }$ & $S U V_{\text {mean }}$ \\
\hline \multicolumn{3}{|l|}{ Young lean } \\
\hline SQUV $_{\max }$ & $\rho=0.79^{\star}$ & \\
\hline$S Q U V_{\text {mean }}$ & & $\rho=0.17$ \\
\hline \multicolumn{3}{|l|}{ Young obese } \\
\hline $\mathrm{SQUV}_{\max }$ & $\rho=0.88^{*}$ & \\
\hline$S Q U V_{\text {mean }}$ & & $\rho=0.58$ \\
\hline \multicolumn{3}{|l|}{ Old lean } \\
\hline SQUV $_{\max }$ & $\rho=0.57$ & \\
\hline$S_{\text {SUV }}$ mean & & $\rho=0.56$ \\
\hline
\end{tabular}

Stratification of Spearman correlations between maximal and mean BAT metabolic activity (respectively, SUV max $_{\text {and }}$ SUV $V_{\text {mean }}$ ) and maximal and mean sympathetic nervous stimulation to BAT (respectively, SQUV $\max$ and $\left.\mathrm{SQUV}_{\text {mean }}\right){ }^{*} P<0.01$. difference between the obese and the lean young subjects. However, other studies, using the same sample size but a different cooling protocol, found a significant difference in metabolic BAT activity and volume between the lean young and the obese $(11,30,31)$.

Likewise, there was no difference in SNS stimulation between the lean and the obese. The strong and positive correlation for detecting BAT on ${ }^{18} \mathrm{~F}$-FDG PET/CT and ${ }^{123} \mathrm{I}-m \mathrm{IBG}$ SPECT/CT we found in the lean young was also found in the obese, confirming that ${ }^{123}$ I- $m$ IBG SPECT/CT is capable of detecting the SNS BAT activity in both the lean young and the obese subjects.

We measured BAT volume using a PET/SPECT threshold-based delineation. Therefore, BAT volumes included in this study reflect activated BAT volume instead of total BAT volume, and total BAT volume might be underestimated. However, by exposing our subjects to cold, BAT is maximally activated. Therefore, in this setting the differences between activated BAT and total BAT are probably small.

We found a weak but positive correlation between energy expenditure during cold exposure and BAT activity, emphasizing that activated BAT could contribute to weight loss. These findings are in line with previous publications $(26,27,32)$.

\section{CONCLUSION}

A lower sympathetic drive may explain the lower BAT activity in the older men but not in the obese men. Furthermore, with increasing age there might also be a diminished sensitivity of BAT to SNS stimulation. If the activation of BAT is regarded as a promising antiobesity strategy, future research has to be directed to identify factors able to reactivate or recruit BAT in thermoneutrality, possibly by increasing the SNS signal to BAT.

\section{DISCLOSURE}

The costs of publication of this article were defrayed in part by the payment of page charges. Therefore, and solely to indicate this fact, this article is hereby marked "advertisement" in accordance with 18 USC section 1734 . No potential conflict of interest relevant to this article was reported.

\section{REFERENCES}

1. Swinburn BA, Sacks G, Hall KD, et al. The global obesity pandemic: shaped by global drivers and local environments. Lancet. 2011;378:804-814.

2. Cunningham SA, Kramer MR, Narayan KM. Incidence of childhood obesity in the United States. N Engl J Med. 2014;370:403-411.

3. Franz MJ, VanWormer JJ, Crain AL, et al. Weight-loss outcomes: a systematic review and meta-analysis of weight-loss clinical trials with a minimum 1-year followup. J Am Diet Assoc. 2007;107:1755-1767.

4. Wing RR, Bolin P, Brancati FL, et al. Cardiovascular effects of intensive lifestyle intervention in type 2 diabetes. $N$ Engl J Med. 2013;369:145-154.

5. Vijgen GH, Sparks LM, Bouvy ND, et al. Increased oxygen consumption in human adipose tissue from the "brown adipose tissue" region. J Clin Endocrinol Metab. 2013;98:E1230-E1234.

6. Blondin DP, Labbé SM, Tingelstad HC, et al. Increased brown adipose tissue oxidative capacity in cold-acclimated humans. J Clin Endocrinol Metab. 2014;99: E438-E446.

7. Nedergaard J, Bengtsson T, Cannon B. Three years with adult human brown adipose tissue. Ann N Y Acad Sci. 2010;1212:E20-E36.

8. Cannon B, Nedergaard J. Brown adipose tissue: function and physiological significance. Physiol Rev. 2004;84:277-359.

9. Cypess AM, Lehman S, Williams G, et al. Identification and importance of brown adipose tissue in adult humans. $N$ Engl J Med. 2009;360:1509-1517.

10. Saito M, Okamatsu-Ogura Y, Matsushita M, et al. High incidence of metabolically active brown adipose tissue in healthy adult humans: effects of cold exposure and adiposity. Diabetes. 2009;58:1526-1531. 
11. van Marken Lichtenbelt WD, Vanhommerig JW, Smulders NM, et al. Coldactivated brown adipose tissue in healthy men. N Engl J Med. 2009;360:15001508 .

12. Yoneshiro T, Aita S, Matsushita M, et al. Age-related decrease in cold-activated brown adipose tissue and accumulation of body fat in healthy humans. Obesity (Silver Spring). 2011;19:1755-1760.

13. Admiraal WM, Holleman F, Bahler L, Soeters MR, Hoekstra JB, Verberne HJ. Combining ${ }^{123}$ I-metaiodobenzylguanidine SPECT/CT and ${ }^{18} \mathrm{~F}-\mathrm{FDG}$ PET/CT for the assessment of brown adipose tissue activity in humans during cold exposure. J Nucl Med. 2013;54:208-212.

14. Admiraal WM, Verberne HJ, Karamat FA, Soeters MR, Hoekstra JB, Holleman F. Cold-induced activity of brown adipose tissue in young lean men of South-Asian and European origin. Diabetologia. 2013;56:2231-2237.

15. Inoue Y, Nakao M, Araki T, Ueda H. Thermoregulatory responses of young and older men to cold exposure. Eur J Appl Physiol Occup Physiol. 1992;65:492-498.

16. Sugarek NJ. Temperature lowering after iced water: enhanced effects in the elderly. J Am Geriatr Soc. 1986;34:526-529.

17. Wagner JA, Horvath SM. Influences of age and gender on human thermoregulatory responses to cold exposures. J Appl Physiol. 1985;58:180-186.

18. Wagner JA, Robinson S, Marino RP. Age and temperature regulation of humans in neutral and cold environments. J Appl Physiol. 1974;37:562-565.

19. Hogikyan RV, Supiano MA. Arterial alpha-adrenergic responsiveness is decreased and SNS activity is increased in older humans. Am J Physiol. 1994;266: E717-E724.

20. Horvath SM, Radcliffe CE, Hutt BK, Spurr GB. Metabolic responses of old people to a cold environment. J Appl Physiol. 1955;8:145-148.

21. Cypess AM, Chen YC, Sze C, et al. Cold but not sympathomimetics activates human brown adipose tissue in vivo. Proc Natl Acad Sci USA. 2012;109:10001-10005.

22. Carey AL, Formosa MF, Van Every B, et al. Ephedrine activates brown adipose tissue in lean but not obese humans. Diabetologia. 2013;56:147-155.
23. Vosselman MJ, van der Lans AA, Brans B, et al. Systemic beta-adrenergic stimulation of thermogenesis is not accompanied by brown adipose tissue activity in humans. Diabetes. 2012;61:3106-3113.

24. Bahler L, Molenaars RJ, Verberne HJ, Holleman F. Role of the autonomic nervous system in activation of human brown adipose tissue: a review of the literature. Diabetes Metab. September 21, 2015 [Epub ahead of print].

25. Cypess AM, Weiner LS, Roberts-Toler C, et al. Activation of human brown adipose tissue by a beta3-adrenergic receptor agonist. Cell Metab. 2015;21:3338.

26. Yoneshiro T, Aita S, Matsushita M, et al. Recruited brown adipose tissue as an antiobesity agent in humans. J Clin Invest. 2013;123:3404-3408.

27. van der Lans AA, Hoeks J, Brans B, et al. Cold acclimation recruits human brown fat and increases nonshivering thermogenesis. J Clin Invest. 2013;123: 3395-3403.

28. Savchenko V, Sung U, Blakely RD. Cell surface trafficking of the antidepressantsensitive norepinephrine transporter revealed with an ectodomain antibody. Mol Cell Neurosci. 2003;24:1131-1150.

29. Bombardieri E, Aktolun C, Baum RP, et al. ${ }^{131} \mathrm{I} /{ }^{123} \mathrm{I}$-metaiodobenzylguanidine (MIBG) scintigraphy: procedure guidelines for tumour imaging. Eur J Nucl Med Mol Imaging. 2003;30:BP132-BP139.

30. Vijgen GH, Bouvy ND, Teule GJ, Brans B, Schrauwen P, van Marken Lichtenbelt WD. Brown adipose tissue in morbidly obese subjects. PLoS One. 2011;6:e17247.

31. Vijgen GH, Bouvy ND, Teule GJ, et al. Increase in brown adipose tissue activity after weight loss in morbidly obese subjects. J Clin Endocrinol Metab. 2012;97: E1229-E1233.

32. Bakker LE, Boon MR, van der Linden RA, et al. Brown adipose tissue volume in healthy lean south Asian adults compared with white Caucasians: a prospective, case-controlled observational study. Lancet Diabetes Endocrinol. 2014;2:210217. 